\title{
REVIEW ARTICLE \\ Mechanisms for floor surfaces or environmental ground contamination to cause human infection: a systematic review
}

\author{
T. RASHID ${ }^{1,2}$, H. VONVILLE ${ }^{2}$, I. HASAN ${ }^{3}$ AND K. W. GAREY ${ }^{1,2 *}$ \\ ${ }^{1}$ University of Houston College of Pharmacy, Houston, TX, USA \\ ${ }^{2}$ UTHealth School of Public Health, Houston TX, USA \\ ${ }^{3}$ University of Edinburgh, Edinburgh, UK
}

Received 12 July 2016; Final revision 31 August 2016; Accepted 31 August 2016;

first published online 26 October 2016

\section{SUMMARY}

Shoe soles have been shown to transfer infectious microorganisms to floor and ground surfaces. However, the possible modes of transmission of infectious agents from floors or ground surfaces to human contact for infection have not been systematically reviewed. A systematic review was performed on articles indexed in medical databases (Medline, EMBASE, PubMed) using a pre-defined search strategy and MeSH terms (date of last search: 15 March 2016). Only primary research studies in English that investigated the transmission dynamics of infectious microorganisms from floor or ground surfaces to human infection were included. Extraction of articles was performed two independent reviewers using pre-defined data fields in an Excel sheet. Disagreements were resolved by consensus. Thirty studies met the inclusion criteria. Almost all hospital-associated microorganisms including methicillin-resistant Staphylococcus aureus, Clostridium difficile, and multidrug-resistant Gram-negative species were identified on floor or ground surfaces. Several modes of transmission dynamics, most commonly direct contact or aerosolization, were identified. In conclusion, interventions such as efficient cleaning of floor surfaces and vectors that transfer infectious organisms to floors such as shoe soles could be an effective infection control strategy to prevent human disease.

Key words: Bacterial infections, Clostridium difficile, disinfection methods, epidemiology, infectious disease transmission, Staphylococcus aureus.

\section{INTRODUCTION}

Shoe soles are almost universally contaminated with infectious microorganisms including many that cause hospital-acquired infection [1, 2]. Our research group has recently reported that up to $40 \%$ of shoe soles in non-healthcare settings are contaminated with toxigenic Clostridium difficile [2]. Several others have

\footnotetext{
* Author for correspondence: K. W. Garey, PharmD, MS, Professor and Chair, University of Houston College of Pharmacy, 1441 Moursund Street, Houston, TX 77030, USA.

(Email: kgarey@uh.edu)
}

shown that shoe soles are a source of contamination of floor surfaces and environmental ground contamination in the hospital and non-hospital setting [3-7]. For example, a study in rural Alaska asked participants to walk on a pre-determined pathway to a local community healthcare centre wearing presterilized boots [8]. Upon entering the building, the participants stepped onto a clean piece of sterilized linoleum. Coliforms including Escherichia coli were present on all boots at the end of the walk and approximately half of the linoleum flooring walked on was also contaminated with these microorganisms. Earlier studies have demonstrated that redistribution 
of bacteria into the air from the operating-room floor accounts for up to $15 \%$ of all airborne bacteria and walking on contaminated floors was a more effective airborne dispersal method than either mopping or sweeping [9]. Despite several apparent mechanisms by which contaminated floors or environmental ground contamination could be a vector for human infections, the transmission dynamics between environmental surfaces and humans have not been systematically reviewed. Thus, this systematic review sets out to assess methods by which floor surfaces or environmental ground contamination can serve as vectors for infectious diseases.

\section{METHODS}

The systematic review used the preferred reporting items of PRISMA to address the study objectives [10]. To be eligible for inclusion, studies had to either directly or indirectly assess infectious microorganism transmission from floor surfaces (hospitals or other healthcare centres) or soil (community) directly to human contact or indirectly to a secondary vector that could transmit the microorganism closer to patient contact. Studies were excluded if they investigated (1) questions not related to modes of transmission of infectious organisms from floors or soil, (2) were infection control or prevention or outbreaks, (3) diversity, geographical distribution, pathology or microbiology of bacterial, viral or fungal agents, (4) genetic or metabolic pathways of infectious agents, (5) disease pathophysiology, diagnosis and treatment in humans, (6) immunization or gene therapies, (7) genetic, modelling, or in vitro research, (8) non-human (plant or animal) infections or (9) were not original research. Only relevant articles published in English between 1946 and 15 September 2016 with available full texts were included in the final review. To maximize the sensitivity to identify relevant articles, the medical databases Medline (1946 to present), PubMed (NLM) and EMBASE (1947 to present) were searched using a broad set of concepts and MeSH terms. MeSH terms included bacteria, virus, fungi, fomites, shoes, disease transmission, reaerosolization, dispersal and dispersion. A complete search strategy for each database (Medline, PubMed, EMBASE) is given in Supplementary Table S1. Scopus (Elsevier) as well as the bibliographies of identified articles were searched for additional studies not found using the initial database search. An auto alert service was set up in Medline (Ovid) for notification of any new articles indexed matching the search terms. An update literature search was done on 15 September 2016 to look for any new related articles being published after the initial database search. Once the search was complete all the citations were uploaded to a citation manager. An online bibliographical management program (Refworks; www.refworks.com/refworks2) was used to manage citations including removal of the internal and external duplicates within and among the databases. A custom MS Excel workbook (Microsoft Corp, USA) for systematic review was designed and used to screen abstracts [11]. Data were abstracted using an Excel file with pre-determined data fields, and was developed, pilot-tested on a random sample of 15 titles, and revised accordingly by the authors. All abstracts and full-text articles were independently reviewed and data were abstracted by two authors (T.R., I.H.) and discrepancies resolved by consensus. Information included author, year of publication, country, study design, study setting, method and findings. Data for findings included source of contamination, contaminant, microbial concentration, measures of association and any other related factors. All data were integrated into a descriptive summary table (Table 1). The primary outcome measure was percentage of microbiological contaminant after contact (directly or indirectly) from floor or ground surfaces.

Quality control during article screening was achieved through (1) a database search conducted by an experienced author, (2) a high Cohen's kappa for agreement between the two authors screening the abstracts, (3) independent searching of all the abstracts and titles by two authors, (4) screeners were blinded to the study author, and (5) independent review of all full-text articles by the two authors. Methodological quality, sample processing, and outcome was used to ascertain the quality of the studies.

\section{RESULTS}

An initial search of Medline, PubMed and EMBASE databases using the key words and MeSH terms yielded 9134 citations. After removal of external and internal duplicates, 5678 unique citations were identified and following screening of the titles and abstracts, 5503 articles were excluded. This yielded 175 full-text articles which were assessed for eligibility and data abstracted; 152 of these did not meet the inclusion and exclusion criteria and were discarded. An additional seven articles which met the selection criteria were identified through bibliographical search 
Table 1. Transmission dynamics from floor surfaces or environmental ground contamination to cause human infection

\begin{tabular}{|c|c|c|c|c|c|c|c|c|c|}
\hline $\begin{array}{l}\text { Study } \\
\text { no. }\end{array}$ & $\begin{array}{l}\text { Transmission } \\
\text { pathway }\end{array}$ & $\begin{array}{l}\text { First author, year, } \\
\text { country }\end{array}$ & $\begin{array}{l}\text { Study } \\
\text { design }\end{array}$ & Setting $(N)$ & Method & $\begin{array}{l}\text { Source of } \\
\text { contamination }\end{array}$ & Contaminant & Measurement & $\begin{array}{l}\text { Predictors of } \\
\text { contamination }\end{array}$ \\
\hline 1 & $\begin{array}{r}\text { Direct via human } \\
\text { contact, hospital }\end{array}$ & $\begin{array}{l}\text { Verde, 2015, } \\
\text { Portugal [12] }\end{array}$ & Obs. & $\begin{array}{l}\text { Three community } \\
\text { hospitals ( } 3177 \\
\text { patients) }\end{array}$ & $\begin{array}{l}\text { Microbiological air } \\
\text { sampling from } \\
\text { hospital areas }\end{array}$ & $\begin{array}{r}\text { Aerosolized floor } \\
\text { microorganisms }\end{array}$ & $\begin{array}{l}\text { Gram-positive cocci } \\
\text { and fungus }\end{array}$ & $\begin{array}{l}\text { Airborne } \\
\text { contamination }\end{array}$ & $\begin{array}{l}\text { Higher traffic areas } \\
\text { and less routine } \\
\text { cleaning predicted } \\
\text { contamination }\end{array}$ \\
\hline 2 & $\begin{array}{r}\text { Direct via human } \\
\text { contact, hospital }\end{array}$ & $\begin{array}{l}\text { Andersson, 2012, } \\
\text { Sweden [13] }\end{array}$ & Obs. & $\begin{array}{l}\text { Hospital operation } \\
\text { room (91 air } \\
\text { samples) }\end{array}$ & $\begin{array}{l}\text { Microbiological air } \\
\text { sampling during } \\
\text { surgery }\end{array}$ & $\begin{array}{r}\text { Aerosolized floor } \\
\text { microorganisms }\end{array}$ & Bacterial pathogens & $\begin{array}{l}\text { Airborne } \\
\text { contamination }\end{array}$ & $\begin{array}{l}\text { Increased } \\
\text { contamination with } \\
\text { person traffic and } \\
\text { duration of surgery }\end{array}$ \\
\hline 3 & $\begin{array}{r}\text { Direct via human } \\
\text { contact, hospital }\end{array}$ & $\begin{array}{l}\text { Anderson, 1982, } \\
\text { USA [15] }\end{array}$ & Obs. & $\begin{array}{l}\text { Paediatric patient } \\
\text { rooms (62 rooms) }\end{array}$ & $\begin{array}{l}\text { Microbiological air } \\
\text { contamination of } \\
\text { carpeting } v s . \text { tiles }\end{array}$ & $\begin{array}{r}\text { Aerosolized floor } \\
\text { microorganisms }\end{array}$ & HAI organisms & $\begin{array}{l}\text { Airborne } \\
\text { contamination }\end{array}$ & $\begin{array}{l}\text { Higher } \\
\text { aerosolization } \\
\text { contamination } \\
\text { above carpeted } \\
\text { flooring }\end{array}$ \\
\hline 4 & $\begin{array}{r}\text { Direct via human } \\
\text { contact, hospital }\end{array}$ & $\begin{array}{l}\text { Limmathurotsakul, } \\
\text { 2013, Thailand } \\
\text { [14] }\end{array}$ & Obs. & $\begin{array}{l}\text { Hospital patients } \\
\text { ( } 286 \text { cases, } 513 \\
\text { controls) }\end{array}$ & $\begin{array}{l}\text { Cases and non- } \\
\text { infected controls } \\
\text { were compared }\end{array}$ & $\begin{array}{l}\text { Soil and water in } \\
\text { hospital } \\
\text { environment }\end{array}$ & $\begin{array}{l}\text { Burkholderia } \\
\text { pseudomallei }\end{array}$ & $\begin{array}{l}\text { Floor } \\
\text { contamination }\end{array}$ & $\begin{array}{l}\text { Exposure to soil or } \\
\text { water increased } \\
\text { melioidosis risk } \\
\text { [OR } 1 \cdot 4(0 \cdot 8-2 \cdot 6)]\end{array}$ \\
\hline 5 & $\begin{array}{r}\text { Direct via human } \\
\text { contact, hospital }\end{array}$ & $\begin{array}{l}\text { Whyte, 2013, } \\
\text { Glasgow [16] }\end{array}$ & Exp. & $\begin{array}{l}\text { Hospital simulation } \\
\text { room (100 steps) }\end{array}$ & $\begin{array}{l}\text { Microbiological air } \\
\text { sampling by human } \\
\text { walking }\end{array}$ & $\begin{array}{r}\text { Aerosolized floor } \\
\text { microorganisms }\end{array}$ & $\mathrm{MCP}$ & $\begin{array}{l}\text { Floor } \\
\text { contamination }\end{array}$ & $\begin{array}{l}\text { Walking significantly } \\
\text { increased MCP } \\
\text { dispersal rate }\end{array}$ \\
\hline 6 & $\begin{array}{r}\text { Direct via human } \\
\text { contact, hospital }\end{array}$ & $\begin{array}{l}\text { Buttner, 2001, USA } \\
\text { [17] }\end{array}$ & Exp. & $\begin{array}{l}\text { Experimental } \\
\text { hospital room ( } 36 \\
\text { surface samples) }\end{array}$ & $\begin{array}{l}\text { Dispersal of } \\
\text { Penicillium } \\
\text { chrysogenum spores } \\
\text { by walking over } \\
\text { carpet and vinyl } \\
\text { floors }\end{array}$ & $\begin{array}{r}\text { Aerosolized floor } \\
\text { microorganisms }\end{array}$ & $\begin{array}{l}\text { P. chrysogenum } \\
\text { spores }\end{array}$ & $\begin{array}{l}\text { Airborne } \\
\text { contamination }\end{array}$ & $\begin{array}{l}\text { Walking caused } \\
\text { similar spore } \\
\text { concentration at } \\
\text { ground level up to } \\
\text { breathing zone }\end{array}$ \\
\hline 7 & $\begin{array}{l}\text { Direct via human } \\
\text { contact, hospital }\end{array}$ & $\begin{array}{l}\text { Gwaltney, 1982, } \\
\text { USA [18] }\end{array}$ & Exp. & $\begin{array}{l}\text { Hospital simulation } \\
\text { room (36 recipients) }\end{array}$ & $\begin{array}{l}\text { Transfer of } \\
\text { rhinovirus- } \\
\text { contaminated floor } \\
\text { tiles to recipients }\end{array}$ & $\begin{array}{l}\text { Floor tile } \\
\text { contamination } \\
\pm \text { antiviral spray }\end{array}$ & Rhinovirus & $\begin{array}{l}\text { Direct human } \\
\text { infection }\end{array}$ & $\begin{array}{c}\text { Higher infection rate } \\
\text { uncleaned }(56 \%) v s \text {. } \\
\text { cleaned }(36 \%) \text { tiles }\end{array}$ \\
\hline 8 & $\begin{array}{l}\text { Direct via human } \\
\text { contact, non- } \\
\text { hospital }\end{array}$ & $\begin{array}{l}\text { Wright, 1968, USA } \\
\text { [19] }\end{array}$ & Obs. & Naval vessels (2) & $\begin{array}{l}\text { Microbiological } \\
\text { contamination of } \\
\text { naval ships }\end{array}$ & $\begin{array}{r}\text { Aerosolized floor } \\
\text { microorganisms }\end{array}$ & Saprophytic bacteria & $\begin{array}{l}\text { Airborne } \\
\text { contamination }\end{array}$ & $\begin{array}{l}\text { Increased } \\
\text { contamination with } \\
\text { person traffic }\end{array}$ \\
\hline 1 & $\begin{array}{l}\text { Direct via } \\
\text { aerosolization, } \\
\text { hospital }\end{array}$ & $\begin{array}{l}\text { Roberts, 2008, UK } \\
\text { [20] }\end{array}$ & Obs. & $\begin{array}{l}\text { Hospital (6-bed } \\
\text { elderly care unit) } \\
\text { and 4-bedded } \\
\text { orthopedic bay } \\
\text { (control) }\end{array}$ & $\begin{array}{l}\text { Dispersal of } \\
\text { C. difficile spores in } \\
\text { patient rooms }\end{array}$ & $\begin{array}{l}\text { Aerosolized } \\
\text { C. difficile from } \\
\text { the hospital } \\
\text { environment }\end{array}$ & C. difficile spores & $\begin{array}{l}\text { Airborne or } \\
\text { surface culture }\end{array}$ & $\begin{array}{l}\text { Aerosolization of } \\
\text { C. difficile present } \\
\text { in all } 23 \text { air samples } \\
\text { during the first } \\
\text { sampling phase and } \\
\text { in none in second } \\
\text { sampling phase }\end{array}$ \\
\hline
\end{tabular}


Table 1 (cont.)

\begin{tabular}{|c|c|c|c|c|c|c|c|c|c|}
\hline $\begin{array}{l}\text { Study } \\
\text { no. }\end{array}$ & $\begin{array}{l}\text { Transmission } \\
\text { pathway }\end{array}$ & $\begin{array}{l}\text { First author, year, } \\
\text { country }\end{array}$ & $\begin{array}{l}\text { Study } \\
\text { design }\end{array}$ & Setting $(N)$ & Method & $\begin{array}{l}\text { Source of } \\
\text { contamination }\end{array}$ & Contaminant & Measurement & $\begin{array}{l}\text { Predictors of } \\
\text { contamination }\end{array}$ \\
\hline 2 & $\begin{array}{l}\text { Direct via } \\
\text { aerosolization, } \\
\text { hospital }\end{array}$ & $\begin{array}{l}\text { Hambraeus, 1978, } \\
\text { Sweden }\end{array}$ & Exp. & $\begin{array}{l}\text { Operation theatre } \\
\text { (movement of } 4 \\
\text { persons) }\end{array}$ & $\begin{array}{l}\text { Dispersal of } S \text {. aureus } \\
\text { bacteria via } \\
\text { walking, wind } \\
\text { currents, and } \\
\text { mopping }\end{array}$ & $\begin{array}{l}\text { Floor } \\
\text { contamination }\end{array}$ & S. aureus & Surface & $\begin{array}{l}\text { Bacteria most widely } \\
\text { dispersed by } \\
\text { walking followed by } \\
\text { increased } \\
\text { ventilation and } \\
\text { mopping }\end{array}$ \\
\hline 3 & $\begin{array}{l}\text { Direct via } \\
\text { aerosolization, } \\
\text { community }\end{array}$ & $\begin{array}{l}\text { Hospodsky, 2012, } \\
\text { USA [22] }\end{array}$ & Obs. & University classroom & $\begin{array}{l}\text { Dispersal of bacterial } \\
\text { genome from } \\
\text { classroom floor dust }\end{array}$ & $\begin{array}{l}\text { Aerosolized floor } \\
\text { dust } \\
\text { microorganisms }\end{array}$ & $\begin{array}{l}\text { Bacterial genome } \\
\text { copy numbers }\end{array}$ & $\begin{array}{l}\text { Airborne } \\
\text { contamination }\end{array}$ & $\begin{array}{l}\text { Human movement } \\
\text { was significantly } \\
\text { associated with } \\
\text { aerosolization of } \\
\text { floor dust } \\
\text { microorganisms }\end{array}$ \\
\hline 4 & $\begin{array}{l}\text { Direct via } \\
\text { aerosolization, } \\
\text { community }\end{array}$ & $\begin{array}{l}\text { Lues, 2006, South } \\
\text { Africa [24] }\end{array}$ & Obs. & Retail outlet & $\begin{array}{l}\text { Dispersal of HAI } \\
\text { bacteria from } \\
\text { randomly selected } \\
\text { delicatessens }\end{array}$ & $\begin{array}{l}\text { Aerosolized } \\
\text { bacteria from } \\
\text { delicatessens }\end{array}$ & $\begin{array}{l}\text { S. aureus and } \\
\text { Enterobacteriaceae }\end{array}$ & Airborne & $\begin{array}{l}\text { Aerosolized bacteria } \\
\text { present in majority } \\
\text { of outlets, increased } \\
\text { with number of } \\
\text { customers }\end{array}$ \\
\hline 5 & $\begin{array}{l}\text { Direct via } \\
\text { aerosolization, } \\
\text { community }\end{array}$ & $\begin{array}{l}\text { Weis, 2002, USA } \\
{[28]}\end{array}$ & Obs. & Office suite & $\begin{array}{l}\text { Dispersal of Bacillus } \\
\text { anthracis spores } \\
\text { from floor dust } \\
\text { based on human } \\
\text { activity }\end{array}$ & $\begin{array}{l}\text { Aerosolized } \\
\text { Bacillus from } \\
\text { office } \\
\text { environment }\end{array}$ & B. anthracis spores & Airborne & $\begin{array}{l}\text { All spores } \\
\text { aerosolized within } \\
10 \text { min. } \\
\text { Aerosolization rate } \\
\text { increased with } \\
\text { human activity }\end{array}$ \\
\hline 6 & $\begin{array}{l}\text { Direct via } \\
\text { aerosolization, } \\
\text { community }\end{array}$ & $\begin{array}{l}\text { Hsing, 2002, USA } \\
\text { [23] }\end{array}$ & Obs. & Office building & $\begin{array}{l}\text { Dispersal of fungal } \\
\text { spores from floor } \\
\text { dust to office chairs }\end{array}$ & $\begin{array}{l}\text { Aerosolized } \\
\text { fungal spores } \\
\text { from floor dust }\end{array}$ & Fungal spores & Surface & $\begin{array}{l}\text { Increased chair } \\
\text { contamination with } \\
\text { increased fungal } \\
\text { spore dust } \\
\text { concentrations }\end{array}$ \\
\hline 7 & $\begin{array}{l}\text { Direct via } \\
\text { aerosolization, } \\
\text { animal worker }\end{array}$ & $\begin{array}{l}\text { O'Connor, 2015, } \\
\text { Australia [21] }\end{array}$ & Obs. & $\begin{array}{l}\text { Sheep yard ( } 25 \\
\text { infected sheep and } \\
45 \text { controls from } \\
\text { another yard) }\end{array}$ & $\begin{array}{l}\text { Sheep yard with Q } \\
\text { fever infected sheep } \\
\text { and controls from } \\
\text { another yard }\end{array}$ & $\begin{array}{l}\text { Aerosolized } \\
\text { ground } \\
\text { microorganisms }\end{array}$ & Coxiella burnetti & $\begin{array}{l}\text { Airborne } \\
\text { contamination }\end{array}$ & $\begin{array}{l}\text { Exposure to } \\
\text { contaminated sheet } \\
\text { yard increased risk } \\
\text { of Q fever [OR } 15 \cdot 3 \\
(P=0 \cdot 014)]\end{array}$ \\
\hline 8 & $\begin{array}{l}\text { Direct via } \\
\text { aerosolization, } \\
\text { animal worker }\end{array}$ & $\begin{array}{c}\text { Brodka, 2012, } \\
\text { Poland [25] }\end{array}$ & Obs. & Poultry house & $\begin{array}{l}\text { Dispersal of } \\
\text { microorganisms at } \\
\text { poultry house to at } \\
\text { least } 1.5 \mathrm{~m}\end{array}$ & $\begin{array}{l}\text { Aerosolized } \\
\text { ground } \\
\text { microorganisms }\end{array}$ & HAI bacteria & $\begin{array}{l}\text { Airborne } \\
\text { contamination }\end{array}$ & $\begin{array}{l}\text { All samples were } \\
\text { positive for } \\
\text { microorganisms at } \\
1.5 \mathrm{~m}\end{array}$ \\
\hline
\end{tabular}


Table 1 (cont.)

\begin{tabular}{|c|c|c|c|c|c|c|c|c|c|}
\hline $\begin{array}{l}\text { Study } \\
\text { no. }\end{array}$ & $\begin{array}{l}\text { Transmission } \\
\text { pathway }\end{array}$ & $\begin{array}{l}\text { First author, year, } \\
\text { country }\end{array}$ & $\begin{array}{l}\text { Study } \\
\text { design }\end{array}$ & Setting $(N)$ & Method & $\begin{array}{l}\text { Source of } \\
\text { contamination }\end{array}$ & Contaminant & Measurement & $\begin{array}{l}\text { Predictors of } \\
\text { contamination }\end{array}$ \\
\hline 9 & $\begin{array}{l}\text { Direct via } \\
\text { aerosolization, } \\
\text { laboratory }\end{array}$ & $\begin{array}{l}\text { Paton, 2015, } \\
\text { UK [44] }\end{array}$ & Exp. & Laboratory setting & $\begin{array}{l}\text { Dispersal of spores } \\
\text { by human walking } \\
\text { over carpet and } \\
\text { PVC flooring }\end{array}$ & $\begin{array}{l}\text { Aerosolized } \\
\text { Bacillus from } \\
\text { office } \\
\text { environment }\end{array}$ & B. anthracis & Airborne & $\begin{array}{l}\text { Type of flooring } \\
\text { (carpet > PVC) and } \\
\text { increased pace of } \\
\text { walking increased } \\
\text { airborne } \\
\text { contamination }\end{array}$ \\
\hline 10 & $\begin{array}{l}\text { Direct via } \\
\text { aerosolization, } \\
\text { laboratory }\end{array}$ & $\begin{array}{l}\text { Rafal, 2002, USA } \\
\text { [26] }\end{array}$ & Exp. & $\begin{array}{l}\text { Aerosolization } \\
\text { chamber }\end{array}$ & $\begin{array}{l}\text { Dispersal of fungal } \\
\text { spores from floor to } \\
\text { ceiling plate }\end{array}$ & $\begin{array}{l}\text { Aerosolization of } \\
\text { fungal spores }\end{array}$ & $\begin{array}{l}\text { Fungal spores and } \\
\text { fragments }\end{array}$ & Airborne & $\begin{array}{l}\text { Aerosolization } \\
\text { increased based on } \\
\text { fungal species, } \\
\text { quantity, air } \\
\text { velocity, and } \\
\text { surface vibration }\end{array}$ \\
\hline 11 & $\begin{array}{l}\text { Direct via } \\
\text { aerosolization, } \\
\text { laboratory }\end{array}$ & $\begin{array}{l}\text { Rafal, 2003, USA } \\
\text { [27] }\end{array}$ & Exp. & $\begin{array}{l}\text { Aerosolization } \\
\text { chamber }\end{array}$ & $\begin{array}{l}\text { Dispersal of } \\
\text { Streptomyces albus } \\
\text { from floor to ceiling } \\
\text { plate }\end{array}$ & $\begin{array}{r}\text { Aerosolization of } \\
\text { Bacterial spores }\end{array}$ & S. albus spores & Airborne & $\begin{array}{l}\text { Aerosolization rate } \\
\text { increases with } \\
\text { increased air } \\
\text { velocity, type and } \\
\text { roughness of surface }\end{array}$ \\
\hline 1 & $\begin{array}{l}\text { Indirect via } \\
\text { arthropods }\end{array}$ & $\begin{array}{l}\text { Aquino, 2011, } \\
\text { Brazil [29] }\end{array}$ & Obs. & $\begin{array}{l}\text { Public hospital } \\
(n=1)\end{array}$ & $\begin{array}{l}\text { Pathogenic soil- } \\
\text { borne fungus } \\
\text { isolated from ants in } \\
\text { hospitals }\end{array}$ & Ants & Fungal spore & Surface & $\begin{array}{l}\text { Pathogenic fungi } \\
\text { isolated from } 40 \% \\
\text { of ants }\end{array}$ \\
\hline 2 & $\begin{array}{l}\text { Indirect via } \\
\text { arthropods }\end{array}$ & $\begin{array}{l}\text { Hsiu-Hua Pai, } \\
\text { 2004, Taiwan [31] }\end{array}$ & Obs. & $\begin{array}{l}\text { Acute care hospitals } \\
(n=90)\end{array}$ & $\begin{array}{l}\text { Cockroaches } \\
\text { cultured for } \\
\text { presence of HAI } \\
\text { microorganisms }\end{array}$ & Cockroaches & HAI pathogens & Surface & $\begin{array}{l}\text { All cockroaches } \\
\text { harboured bacteria } \\
\text { with resistance to at } \\
\text { least one antibiotic }\end{array}$ \\
\hline 3 & $\begin{array}{l}\text { Indirect via } \\
\text { arthropods }\end{array}$ & $\begin{array}{l}\text { Fotedar, 1993, } \\
\text { India [32] }\end{array}$ & Obs. & $\begin{array}{l}\text { Surgical ward of } \\
\text { hospital }\end{array}$ & $\begin{array}{l}\text { Cockroaches from } \\
\text { surgical ward } \\
\text { cultured for HAI } \\
\text { organisms }\end{array}$ & Cockroaches & HAI pathogens & Surface & $\begin{array}{c}\text { Almost all }(>90 \%) \\
\text { cockroaches had } \\
\text { HAI pathogens }\end{array}$ \\
\hline 4 & $\begin{array}{l}\text { Indirect via } \\
\text { arthropods }\end{array}$ & $\begin{array}{l}\text { Sramova, 1991, } \\
\text { Czechoslovakia } \\
\text { [30] }\end{array}$ & Obs. & $\begin{array}{l}\text { Healthcare facilities } \\
(n=55)\end{array}$ & $\begin{array}{l}\text { Arthropods cultured } \\
\text { for HAI pathogens }\end{array}$ & Arthropods* & HAI pathogens & Surface & $\begin{array}{l}\text { Contamination rate: } \\
13-59 \% \text {. Higher } \\
\text { rates with increased } \\
\text { arthropod mobility }\end{array}$ \\
\hline 5 & $\begin{array}{l}\text { Indirect via } \\
\text { arthropods }\end{array}$ & $\begin{array}{l}\text { Sola-Gines, 2015, } \\
\text { Spain [33] }\end{array}$ & Obs. & $\begin{array}{l}\text { Chicken broiler } \\
\text { farms }(n=5)\end{array}$ & $\begin{array}{l}\text { Flies cultured for } \\
\text { ESBL E. coli }\end{array}$ & Flies & $\begin{array}{l}\text { Cephalosporin- } \\
\text { resistant E. coli }\end{array}$ & Surface & $\begin{array}{l}42 \text { of } 682(6 \cdot 2 \%) \text { flies } \\
\text { contaminated }\end{array}$ \\
\hline 1 & $\begin{array}{l}\text { Indirect, other } \\
\text { mechanisms }\end{array}$ & $\begin{array}{l}\text { Jonges, 2015, } \\
\text { Netherlands [45] }\end{array}$ & Obs. & Poultry farms & $\begin{array}{l}\text { Particulate matter or } \\
\text { inhalable dust } \\
\text { particles around } \\
\text { poultry farms }\end{array}$ & $\begin{array}{l}\text { Wind-mediated } \\
\text { dispersal }\end{array}$ & $\begin{array}{l}\text { Avian Influenza } \\
\text { Virus }\end{array}$ & Surface & $\begin{array}{l}\text { Influenza virus } \\
\text { detected up to } 60 \mathrm{~m} \\
\text { downwind from the } \\
\text { barn }\end{array}$ \\
\hline
\end{tabular}


Table 1 (cont.)

\begin{tabular}{|c|c|c|c|c|c|c|c|c|c|}
\hline $\begin{array}{l}\text { Study } \\
\text { no. }\end{array}$ & $\begin{array}{l}\text { Transmission } \\
\text { pathway }\end{array}$ & $\begin{array}{l}\text { First author, year, } \\
\text { country }\end{array}$ & $\begin{array}{l}\text { Study } \\
\text { design }\end{array}$ & Setting $(N)$ & Method & $\begin{array}{l}\text { Source of } \\
\text { contamination }\end{array}$ & Contaminant & Measurement & $\begin{array}{l}\text { Predictors of } \\
\text { contamination }\end{array}$ \\
\hline 2 & $\begin{array}{l}\text { Indirect, other } \\
\text { mechanisms }\end{array}$ & $\begin{array}{l}\text { Monaghan, } \\
\text { 2012, UK [36] }\end{array}$ & Obs. & Community & $\begin{array}{l}\text { E. coli marker } \\
\text { bacteria inoculated } \\
\text { in irrigation water }\end{array}$ & Soil splash & E. coli & Surface & $\begin{array}{l}\text { Irrigation water } \\
\text { splash disperse } \\
\text { bacteria at least } 25 \\
\text { cm horizontally } 20 \\
\text { cm high }\end{array}$ \\
\hline 3 & $\begin{array}{l}\text { Indirect, other } \\
\text { mechanisms }\end{array}$ & $\begin{array}{l}\text { Nieuwenhuijsen, } \\
\text { 1999, USA [37] }\end{array}$ & Obs. & Farms $(n=10)$ & $\begin{array}{l}\text { Workers tested for } \\
\text { inhalable and } \\
\text { respirable } \\
\text { endotoxin } \\
\text { concentration }\end{array}$ & $\begin{array}{l}\text { Aerosolization } \\
\text { and inhalation } \\
\text { of endotoxin via } \\
\text { farm activities }\end{array}$ & $\begin{array}{l}\text { Dust and bacterial } \\
\text { endotoxin }\end{array}$ & Aerosolization & $\begin{array}{l}\text { Cleaning of poultry } \\
\text { houses associated } \\
\text { with highest levels } \\
\text { of endotoxin }\end{array}$ \\
\hline 4 & $\begin{array}{l}\text { Indirect, other } \\
\text { mechanisms }\end{array}$ & $\begin{array}{l}\text { Gagniere, } \\
\text { 2006, France [35] }\end{array}$ & Exp. & Food plant & $\begin{array}{l}\text { High-pressure water } \\
\text { cleaning of surfaces } \\
\text { of food industry }\end{array}$ & $\begin{array}{l}\text { High-pressure } \\
\text { aerosolization }\end{array}$ & Pseudomonas putida & Surface & $\begin{array}{l}\text { P. putida adherence } \\
\text { to high-pressure } \\
\text { water pipe. }\end{array}$ \\
\hline 5 & $\begin{array}{l}\text { Indirect, other } \\
\text { mechanisms }\end{array}$ & $\begin{array}{l}\text { DiCaprio, 2015, } \\
\text { USA [34] }\end{array}$ & Exp. & Farm soil & $\begin{array}{l}\text { Strawberry plants } \\
\text { cultured after } \\
\text { planting in virus- } \\
\text { contaminated soil }\end{array}$ & $\begin{array}{l}\text { Contaminated } \\
\text { soil }\end{array}$ & $\begin{array}{l}\text { Murine norovirus-1 } \\
\text { (MNV-1) and } \\
\text { Tulane virus (TV) }\end{array}$ & Surface & $\begin{array}{l}\text { Virus present on } 33 \% \\
\text { of strawberries }\end{array}$ \\
\hline 6 & $\begin{array}{l}\text { Indirect, other } \\
\text { mechanisms }\end{array}$ & $\begin{array}{l}\text { Penet, 2014, France } \\
\text { [38] }\end{array}$ & Exp. & Community & $\begin{array}{l}\text { Dispersal of fungal } \\
\text { spores by rain } \\
\text { splash }\end{array}$ & $\begin{array}{l}\text { Rain splash } \\
\text { dispersal }\end{array}$ & $\begin{array}{l}\text { Colletotrichum } \\
\text { gloeosporioides }\end{array}$ & Surface & $\begin{array}{l}\text { Direct splashing } \\
\text { contributed to } \\
\text { dispersal of fungal } \\
\text { spores }\end{array}$ \\
\hline
\end{tabular}

Obs., Observational; Exp, experimental; HAI, healthcare-associated infection; OR, odds ratio; MCP, microbe-containing particle; ESBL, extended-spectrum beta-lactamase.

* Arthropods included cockroaches, ants, and other insects. 

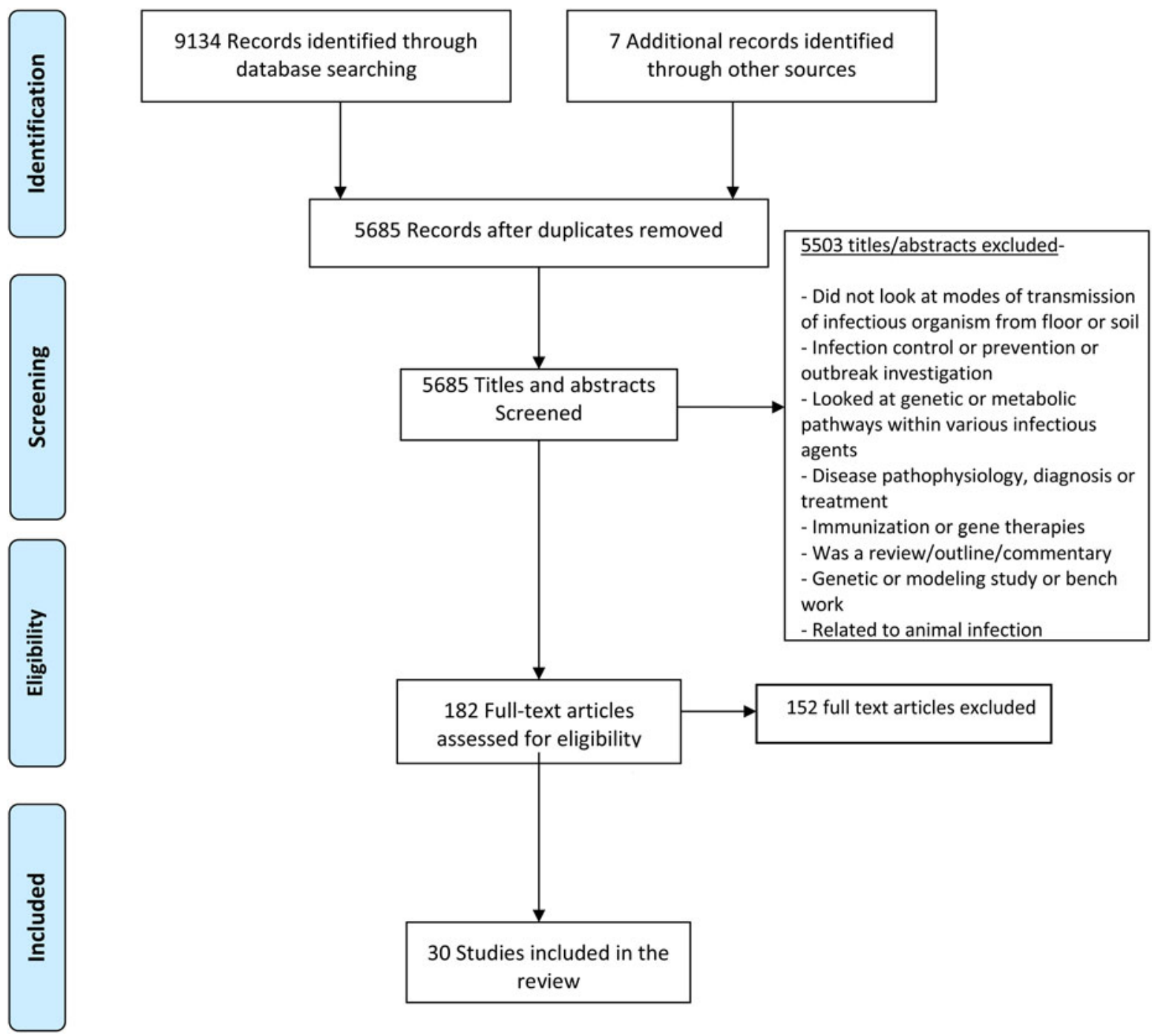

Fig. 1. PRISMA flowchart for screening and evaluation of studies looking at transmission dynamics.

of the selected articles and were included in the review, giving a final total of 30 articles which met all study criteria (Table 1). A Cohen's kappa of agreement between the two reviewers was $96 \%$ [Cohen's kappa for inter rater reliability $\kappa=0.96]$. The detailed article selection process is outlined in the PRISMA [Preferred Reporting Items for Systemic Reviews] flowchart in Figure 1. Methods of disease transmission were categorized as direct or indirect pathways. Direct pathways included the transmission mechanisms resulting in direct contact of human beings with microorganisms on floor or ground surfaces. Indirect pathways were defined as mechanisms that were not directly related to human contact of floor or ground surfaces but included vectors that could lead to human infection via a secondary transmission event. Of the 30 articles (published between 1968 and 2015) included in the review, 19 examined direct pathways of transmission and 11, indirect pathways; 20 were observational and 10 were experimental studies. Most studies were from the USA or Europe.

\section{Direct pathways of transmission}

\section{Direct human contact}

Identified direct pathways included human activities directly related to touching of floor surfaces or aerosolization from contaminated floor surfaces to contact humans. Eight of nine direct human contact studies involved an actual or simulated hospital setting. A number of variables were associated with airborne microorganism contamination including number of visitors and human activities to a patient's room [12], 
number of persons and person movement in the operating room [13], and presence of dirt (either soil or water) in hospital areas [14]. Carpets also yielded higher microbial concentrations per square inch compared to bare floors in a paediatric patient's room [15].

Experimental studies investigated walking over contaminated flooring (two studies) or floor cleaning to prevent infection. Both studies that assessed walking identified increased contamination of either the surrounding floor [16] or airborne contamination [17] and varied by the type of flooring with cut pile carpet showing greater contamination than vinyl and loop pile carpet. Another experimental study [18] demonstrated reduced numbers of microorganisms on the finger tips following touching of artificially contaminated untreated tiles $(61 \%)$ compared to tiles treated with an antiviral compound (21\%). Finally, an observational study undertaken on two naval vessels identified a direct positive association between personnel concentration with bacterial concentration [19]. Thus, all human contact studies showed that activities such as walking, touching or contact with floor surfaces are important factors for dispersal of pathogenic microorganisms.

\section{Aerosolization}

Eleven studies investigated transmission of floor or ground microorganisms by aerosolization. Two of the studies were performed in hospitals, four in the community, two in animal workers, and three in the laboratory. Aerosolization methods included airborne dispersal, and aerial and dust dissemination. In the hospital studies sporadic airborne dissemination of $C$. diffcile in the hospital environment was identified during routine cleaning procedures and aerosolization was linked with the persistence of the bacteria on environmental surfaces [20]. Hambraeus et al. [9] investigated various modes of airborne dispersal of contaminated hospital-floor bacteria and showed that walking led to the highest dispersal of Staphylococcus aureus and other microorganisms at levels three-fold higher than found with heating, ventilation and air conditioning (HVAC) ventilation, and 17-fold higher than with floor mopping. Active air movement in the room (HVAC) increased persistence of bacterial particles in the environment from $1.9 \mathrm{~h}$ without ventilation to $14 \cdot 3 \mathrm{~h}$ with ventilation. These results were corroborated by three community-based studies [21-25] in which airborne dispersal of pathogenic bacteria was identified in a university classroom, retail outlets, and office suites, and all studies recorded increased human movement as significant predictors of contamination. Notably, two of the retail outlet studies found the airborne bacterial load to be significantly related to the number of visitors attending the outlet $[24,25]$.

Three experimental studies investigated the effect of flooring type on aerosolization $(n=1)$, and vertical dispersal of fungal or bacterial spores $(n=2)$ [26-28]. Carpet was more likely to cause aerosolization than PVC flooring along with increased traffic of persons over the flooring. Aerosolization probability increased with higher air velocity, type and texture of surfaces, and number, size and type of microorganism. Greater than $80 \%$ of aerosolized bacterial particles were within the alveolar respirable size [28].

\section{Indirect pathways of transmission}

\section{Arthropod-borne transmission}

Five observational studies (four in hospitals) investigated arthropod-borne transmission of microorganisms [29-32] and in chicken broiler farms [33]. The hospitalbased studies were performed in Brazil, Taiwan, India, and Czechoslovakia and examined the presence of healthcare-acquired organisms on the surfaces of arthropods. Healthcare-associated pathogens were identified in arthropods in all studies. Arthropod- and cockroach-borne transmission of bacteria was linked in two studies with nosocomial infection and spread of multidrug-resistant organisms [31, 32]. In the community setting, flies in broiler houses were found to be contaminated with genetically identical E. coli strains producing extended-spectrum beta-lactamases which were also present in the farm environment [33].

Six studies investigated indirect pathways of transmission of environmental microorganisms from plants, water, and soil to human infection. For example, strawberries grown in virus-contaminated soil resulted in internalization of the virus into the internal surface of the strawberry and it was postulated that the virus could then enter the human food chain and cause infection despite adequate washing of the external surface prior to eating [34]. Rain splash due to irrigation was also shown to cause local dispersal of contaminated soil to crops or other fresh produce [35-38].

\section{DISCUSSION}

Transmission dynamics of infectious diseases from the environment are complex. Our research group has previously demonstrated that shoe soles are often highly contaminated with $C$. difficile [2], a finding 
Table 2. Summary of transmission pathways

\begin{tabular}{|c|c|c|c|c|}
\hline $\begin{array}{l}\text { Pathways of } \\
\text { transmission }\end{array}$ & $\begin{array}{l}\text { Modes of } \\
\text { transmission } \\
\text { (studies) }\end{array}$ & Variables investigated & Organisms & Comments \\
\hline \multirow[t]{4}{*}{$\begin{array}{l}\text { Direct } \\
\text { pathways }\end{array}$} & $\begin{array}{l}\text { Aerosolization } \\
(n=13)\end{array}$ & $\begin{array}{l}\text { - Reaerosolization } \\
\text { - Aerosolization of } \\
\text { respirable size particles } \\
\text { - Wind and airborne } \\
\text { dispersal } \\
\text { - Dust dispersal } \\
\text { - Redispersal factors } \\
\text { - Decay rate for bacteria } \\
\text { carrying particles } \\
\text { - High-pressure dispersal }\end{array}$ & $\begin{array}{l}\text { - Bacillus anthracis } \\
\text { - Clostridium difficile } \\
\text { - Staphylococcus } \\
\text { - } \text { aureus } \\
\text { - Enterobacteriaceae } \\
\text { - Escherichia coli } \\
\text { - Klebsiella } \\
\text { pneumoniae } \\
\text { - Pseudomonas } \\
\text { - Streptomyces } \\
\text { - Fungal spores }\end{array}$ & $\begin{array}{l}\text { - Walking identified as one of } \\
\text { the most important redispersal } \\
\text { factors } \\
\text { - Aerosolization depended on } \\
\text { type of surface, presence of } \\
\text { carpeting, number and size of } \\
\text { particles, air velocity } \\
\text { - Many aerosolized particles are } \\
\text { in respirable range }\end{array}$ \\
\hline & Human contact & & & \\
\hline & $\begin{array}{l}\text { Direct touch } \\
(n=1)\end{array}$ & $\begin{array}{l}\text { - Transfer of particles by } \\
\text { touching the floor } \\
\text { surfaces }\end{array}$ & - Rhinovirus & $\begin{array}{l}\text { - Appropriate disinfection of } \\
\text { floor surface decreased } \\
\text { infection rates }\end{array}$ \\
\hline & $\begin{array}{l}\text { Human activities } \\
(n=8)\end{array}$ & $\begin{array}{l}\text { Human movement } \\
\text { including, walking, } \\
\text { mopping, blowing, } \\
\text { traffic flow }\end{array}$ & $\begin{array}{l}\text { - Burkholderia } \\
\text { pseudomallei } \\
\text { - Penicillium } \\
\text { chrysogenum } \\
\text { - Saprophytic bacteria } \\
\text { - Gram-positive cocci }\end{array}$ & $\begin{array}{l}\text { Human activities including } \\
\text { walking were important factor } \\
\text { for transmission of floor } \\
\text { bacteria }\end{array}$ \\
\hline \multirow[t]{4}{*}{$\begin{array}{l}\text { Indirect } \\
\text { pathways }\end{array}$} & $\begin{array}{l}\text { Arthropod-borne } \\
(n=5)\end{array}$ & $\begin{array}{l}\text { - Cockroaches, flies, other } \\
\text { arthropods }\end{array}$ & $\begin{array}{l}\text { - Gram-positive and } \\
\text { Gram-negative } \\
\text { bacteria } \\
\text { - Fungus }\end{array}$ & $\begin{array}{l}\text { Various arthropods harbour } \\
\text { clinically important resistant } \\
\text { bacteria and fungi }\end{array}$ \\
\hline & Other mechanisms & & & \\
\hline & Foodborne $(n=3)$ & $\begin{array}{l}\text { - Contaminated soil } \\
\text { - Rain splash } \\
\text { - Soil splash }\end{array}$ & $\begin{array}{l}\text { - Murine norovirus } \\
\text { - Fungi } \\
\text { - Escherichia coli }\end{array}$ & $\begin{array}{l}\text { Plants grown in contaminated } \\
\text { soil results can internalize } \\
\text { organisms and enter food } \\
\text { chain }\end{array}$ \\
\hline & $\begin{array}{l}\text { Waterborne } \\
(n=1)\end{array}$ & - High-pressure dispersal & - Pseudomonas putida & $\begin{array}{l}\text { High-pressure dispersal of } \\
\text { floor bacteria to nozzles }\end{array}$ \\
\hline
\end{tabular}

concordant with several other studies showing the presence of pathogenic microorganisms on shoe soles $[2,39]$. Such contamination can readily spread to the floor or ground surface through walking and so we decided to assess possible modes of transmission of infectious agents from such surfaces to human contact to increase the likelihood of human infection. This was achieved through a systematic literature review and to our knowledge this is the first such review to do so. After identifying 30 separate studies, we grouped transmission dynamics into two pathways: (1) that would directly increase the likelihood of human infection or (2) indirectly increase the likelihood via a secondary vector. Studies performed in hospitals, community, and animal farms have all demonstrated the increased likelihood of human infection either via direct contact with the floor surface or via aerosolization (Table 2) as walking over contaminated floors has consistently been shown to aerosolize infectious particles to a height at which respiration of the organism would be possible.

Despite a large amount of epidemiological and experimental evidence, very few interventional studies were identified but almost all studies demonstrated that increased human movement with walking increased the likelihood of airborne dispersal of microorganisms. This suggests that effective disinfection and cleaning of floors and shoe soles could directly impact on aerosol generation rates. This is supported by Gwaltney \& Hendley [18] who found that cleaning of floors contaminated with a rhinovirus with a phenol/ alcohol disinfectant significantly reduced both the recovery of the virus on the surface and the rate of detection of virus on fingers, as well as transmission and 
infection of volunteer recipients. For effective antimicrobial cleaning, a broad spectrum disinfecting agent would be required as studies have shown a very wide range of microorganisms including many with multi-drug resistance. This systematic review also suggests that targeted interventions in high-traffic area would likely have the highest probability of decreasing the likelihood of human infection via direct contact or aerosolization from a microbially contaminated environment. Indeed, in rooms contaminated with multidrug-resistant Acinetobacter baumannii, it has been repeatedly shown that up to $25 \%$ of rooms may remain contaminated with the organism despite apparent appropriate terminal cleaning [40]. Moreover, a recent experimental study using a non-pathogenic viral surrogate marker to assess floor contamination also demonstrated the potential of hospital floors to be a source of pathogen dissemination [41]. Taken together, these findings suggest that there is a high likelihood that contaminated shoe soles entering these rooms shortly after terminal cleaning result in re-contamination of the room. This hypothesis will require future study. According to recent studies, use of novel probiotic-based cleaning interventions or customized ultraviolet radiation disinfection devices may be more effective than chemical disinfection in cleaning rooms of pathogenic organisms [42, 43].

The strengths of this study include state-of-the-art systematic review techniques including multiple database searches, multiple independent reviewers, and reproducible results, which points the way to future intervention studies. The limitations are that the search strategy was limited to articles identified from Ovid Medline, PubMed and EMBASE and some topic-related articles indexed in other databases may have been missed. Further, studies were heterogeneous in design with regard to sampling strategy, population surveyed, and disinfecting practice, making a meta-analysis of the data not possible. Nevertheless, a consistently high rate of environmental contamination was noted in all studies and future studies are warranted to understand better the probability and frequency of microbial transfer from shoes to flooring surfaces or other areas that impact on the disease transmission model. Certainly, an effective disinfection strategy for shoe soles is urgently needed.

In conclusion, this study identified direct and indirect routes of transmission of infectious microorganisms from floor or ground surfaces to human infection. Interventions including cleaning of floor surfaces and identification of vectors that transfer such organisms such as shoe soles could constitute part of an effective infection control strategy in hospital and community settings.

\section{SUPPLEMENTARY MATERIAL}

For supplementary material accompanying this paper visit http://dx.doi.org/10.1017/S0950268816002193.

\section{ACKNOWLEDGEMENTS}

This work was supported by a research grant from Healthy Sole LLC.

\section{DECLARATION OF INTEREST}

None.

\section{REFERENCES}

1. Agarwal M, Hamilton-Stewart P, Dixon RA. Contaminated operating room boots: the potential for infection. American Journal of Infection control 2002; 30: 179-183.

2. Alam MJ, et al. Investigation of potentially pathogenic Clostridium difficile contamination in household environs. Anaerobe 2014; 27: 31-33.

3. Amass SF, et al. Effectiveness of using a mat filled with a peroxygen disinfectant to minimize shoe sole contamination in a veterinary hospital. Journal of the American Veterinary Medical Association 2006; 228: 1391-1396.

4. Amirfeyz R, et al. Theatre shoes - a link in the common pathway of postoperative wound infection? Annals of the Royal College of Surgeons of England 2007; 89: 605-608.

5. Ayliffe GA, Collins BJ, Lowbury EJ. Cleaning and disinfection of hospital floors. British Medical Journal 1966; 2: 442-445.

6. Curry CH, et al. Could tourist boots act as vectors for disease transmission in Antarctica? Journal of Travel Medicine 2002; 9: 190-193.

7. Eisenberg T, et al. Boot swabs to collect environmental samples from common locations in dairy herds for Mycobacterium avium ssp. paratuberculosis (MAP) detection. Journal of Dairy Research 2013; 80: 485-489.

8. Chambers MK, et al. Transport of fecal bacteria by boots and vehicle tires in a rural Alaskan community. Journal of Environmental Management 2009; 90: 961-966.

9. Hambraeus A, Bengtsson S, Laurell G. Bacterial contamination in a modern operating suite. 3. Importance of floor contamination as a source of airborne bacteria. Journal of Hygiene (London) 1978; 80: 169-174.

10. Moher D, et al. Preferred reporting items for systematic reviews and meta-analyses: the PRISMA statement. Annals of Internal Medicine 2009; 151: 264-269.

11. UTHealth. Excel workbooks for systematic reviews, 2016 (http://libguides.sph.uth.tmc.edu/excel_SR_work book). Accessed 31 August 2016.

12. Verde SC, et al. Microbiological assessment of indoor air quality at different hospital sites. Research in Microbiology 2015; 166: 557-563. 
13. Andersson AE, et al. Traffic flow in the operating room: An explorative and descriptive study on air quality during orthopedic trauma implant surgery. American Journal of Infection Control 2012; 40: 750-755.

14. Limmathurotsakul D, et al. Activities of daily living associated with acquisition of melioidosis in northeast Thailand: a matched case-control study. PLoS Neglected and Tropical Diseases 2013; 7: e2072.

15. Anderson RL, et al. Carpeting in hospitals: an epidemiological evaluation. Journal of Clinical Microbiology 1982; 15: 408-415.

16. Whyte W, et al. Dispersion of microbes from floors when walking in ventilated rooms. International Journal of Ventilation 2013; 12: 271-284.

17. Buttner MP, et al. Measurement of airborne fungal spore dispersal from three types of flooring materials. Aerobiologia 2002; 18: 1-11.

18. Gwaltney JM, Hendley JO. Transmission of experimental rhinovirus infection by contaminated surfaces. American Journal of Epidemiology 1982; 116: 828-833.

19. Wright D, Vaichulis E, Chatigny M. Biohazard determination of crowded living-working spaces: airborne bacteria aboard two naval vessels. American Industrial Hygiene Association Journal 1968; 29: 574-581.

20. Roberts K, et al. Aerial dissemination of Clostridium difficile spores. BMC Infectious Disease 2008; 8: 7.

21. O'Connor B, Tribe IG, Givney R. A windy day in a sheep saleyard: an outbreak of Q fever in rural South Australia. Epidemiology and Infection 2015; 143: 391-398.

22. Hospodsky D, et al. Human occupancy as a source of indoor airborne bacteria. PLoS ONE 2012; 7: e34867.

23. Chao HJ, et al. Dustborne fungi in large office buildings. Mycopathologia 2002; 154: 93-106.

24. Lues JF, Theron MM, Van Tonder I. Food-associated bacteria in bioaerosols of delicatessens. International Journal of Environmental and Health Research 2006; 16: 419-426.

25. Bródka K, et al. The variability of bacterial aerosol in poultry houses depending on selected factors. International Journal of Occupational Medicine and Environmental Health 2012; 25: 281-293.

26. Górny RL, Dutkiewicz J. Bacterial and fungal aerosols in indoor environment in Central and Eastern European countries. Annals of Agricultural and Environmental Medicine 2002; 9: 17-23.

27. Górny RL, et al. Release of Streptomyces albus propagules from contaminated surfaces. Environmental Research 2003; 91: 45-53.

28. Weis CP, et al. Secondary aerosolization of viable Bacillus anthracis spores in a contaminated US Senate Office. Journal of the American Medical Association 2002; 288: 2853-2858.

29. Aquino R, et al. Filamentous fungi vectored by ants (Hymenoptera: Formicidae) in a public hospital in north-eastern Brazil. Journal of Hospital Infection 2013; 83: 200-204.

30. Šrámová $\mathbf{H}$, et al. Epidemiological role of arthropods detectable in health facilities. Journal of Hospital Infection 1992; 20: 281-292.
31. Pai HH, Chen WC, Peng CF. Cockroaches as potential vectors of nosocomial infections. Infection Control and Hospital Epidemiology 2004; 25: 979-984.

32. Fotedar R, Banerjee U. Vector potential of the German cockroach in dissemination of Pseudomonas aeruginosa. Journal of Hospital Infection 1993; 23: 55-59.

33. Solà-Ginés M, et al. Houseflies (Musca domestica) as vectors for extended-spectrum $\beta$-lactamase-producing Escherichia coli on Spanish broiler farms. Applied Environmental Microbiology 2015; 81: 3604-3611.

34. DiCaprio E, Culbertson D, Li J. Evidence of the internalization of animal caliciviruses via the roots of growing strawberry plants and dissemination to the fruit. Applied Environmental Microbiology 2015; 81: 2727-2734.

35. Gagniere S, Auvray F, Carpentier B. Spread of a green fluorescent protein-tagged Pseudomonas putida in a water pipe following airborne contamination. Journal of Food Protein 2006; 69: 2692-2696.

36. Monaghan J, Hutchison M. Distribution and decline of human pathogenic bacteria in soil after application in irrigation water and the potential for soil-splash-mediated dispersal onto fresh produce. Journal of Applied Microbiology 2012; 112: 1007-1019.

37. Nieuwenhuijsen MJ, et al. Personal exposure to dust, endotoxin and crystalline silica in California agriculture. Annals of Occupational Hygiene 1999; 43: 35-42.

38. Penet $\mathbf{L}$, et al. Direct splash dispersal prevails over indirect and subsequent spread during rains in Colletotrichum gloeosporioides infecting yams. PLoS ONE 2014; 9: e115757.

39. Rashid T, et al. Shoe soles as a potential vector for pathogen transmission: a systematic review. Journal of Applied Microbiology. Published online 6 August 2016. doi:10.1111/jam.13250.

40. Strassle P, et al. The effect of terminal cleaning on environmental contamination rates of multidrugresistant Acinetobacter baumannii. American Journal of Infection Control 2012; 40.

41. Koganti S, et al. Evaluation of hospital floors as a potential source of pathogen dissemination using a nonpathogenic virus as a surrogate marker. Infection Control and Hospital Epidemiology. Published online 15 August 2016. doi:10.1017/ice.2016.181.

42. Caselli E, et al. Impact of a probiotic-based cleaning intervention on the microbiota ecosystem of the hospital surfaces: focus on the resistome remodulation. PLoS ONE 2016; 11: e0148857.

43. Mathew JI, et al. Evaluation of an enclosed ultraviolet-C radiation device for decontamination of mobile handheld devices. American Journal of Infection Control 2016; 44: 724-726.

44. Paton S, et al. Reaerosolization of spores from flooring surfaces to assess the risk of dissemination and transmission of infections. Applied and Environmental Microbiology 2015; 81: 4914-4919.

45. Jonges M, et al. Wind-mediated spread of lowpathogenic avian influenza virus into the environment during outbreaks at commercial poultry farms. PLoS ONE 2015; 10: e0125401. 